\title{
Hérnia medular idiopática atípica - relato de caso
}

\section{Atypical Idiopathic Spine Cord Herniation (ISCH) - Case Report}

\author{
Heber Martim Vieira ${ }^{1}$ Rodrigo Amaral ${ }^{1}$ Luis Marchi ${ }^{1}$ Gabriel Pokorny ${ }^{1}$ Fernando Marcelino ${ }^{1}$ \\ Fabio Rosa ${ }^{1}$ Angelo Guarçoni Netto ${ }^{1}$ Nicholai Faulhaber ${ }^{1}$ Rubens Jensen ${ }^{1}$ Luiz Pimenta ${ }^{1}$ \\ ${ }^{1}$ Instituto de Patologia da Coluna (IPC), São Paulo, SP, Brasil \\ Arq Bras Neurocir 2018;37:123-127. \\ Address for correspondence Gabriel Pokorny, BSc, Instituto de \\ Patologia da Coluna (IPC), R. Vergueiro, 1421, São Paulo, SP, Brazil \\ (e-mail: g.pokorny@patologiadacoluna.com.br).
}

\section{Resumo \\ Palavras-Chave \\ - coluna vertebral \\ - medula espinhal \\ - canal vertebral \\ - hérnia \\ - hérnia medular \\ - microcirurgia}

A hérnia medular idiopática (HMI) é uma causa rara de mielopatia progressiva que afeta principalmente mulheres de meia idade com apresentação clínica típica com a Síndrome de Brown-Sequard. Possui etiologia incerta, sendo a teoria mais aceita a de ser um defeito congênito na dura-máter que leva a uma herniação lenta e progressiva da medula que ocasiona uma lesão evolutiva, podendo levar a um déficit irreversível quando subdiagnosticado e não tratado da forma ideal. A realização da ressonância magnética é fundamental para o diagnóstico, e a cirurgia é o tratamento de escolha para reverter e cessar os sintomas mielopáticos. O presente artigo mostra um caso de uma apresentação atípica da localização do defeito dural e da herniação, não descrita ainda na literatura, levando a uma apresentação neurológica e anatômica incomum para esta patologia, obrigando a realizar um planejamento cirúrgico específico para tal caso.

Idiopathic medullary hernia is a rare cause of progressive myelopathy, primarily affecting middle-aged women, typical clinical presentation with Brown-Sequárd Syndrome. Its etiology is uncertain, but the most accepted theory is that a congenital defect in the dura mater leads to a slow and progressive spinal cord herniation, causing an evolutionary spinal cord injury, which can lead to an irreversible deficit when underdiagnosed and not treated adequately. Magnetic resonance imaging is essential for the diagnosis, and surgery is the treatment of choice to reverse and stop myelopathy symptoms. The present article shows a case of an atypical presentation of the location of the dural defect and herniation, not yet described in the literature, leading to an unusual neurologic and anatomical presentation for this pathology, requiring a specific planning for this case.

\section{Introdução}

Hérnia medular idiopática (HMI) é uma condição rara de mielopatia, que acomete tipicamente pacientes do sexo feminino de meia idade. Essa condição foi descrita pela primeira vez por Wortzman em $1974,{ }^{1}$ e teve menos de 174 casos descritos na literatura até $2016 .^{2}$ A HMI consiste em um defeito de fechamento dos folhetos da dura-máter, mais frequentemente em sua porção ventral, resultando em uma herniação do conteúdo medular, podendo ser de natureza congênita ou adquirida, provocada por trauma ou pósprocedimento cirúrgico, mas sua patogênese ainda é incerta. A história habitual da doença é de uma mielopatia de evolução lenta e progressiva, com a apresentação clínica da received

February 26, 2018

accepted

April 3, 2018

published online

May 18, 2018
DOI https://doi.org/

10.1055/s-0038-1655745. ISSN 0103-5355.
Copyright (c) 2018 by Thieme Revinter

Publicações Ltda, Rio de Janeiro, Brazil
License terms

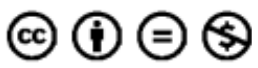


síndrome de Brown-Sequard na grande maioria dos casos. $\mathrm{O}$ Diagnóstico da Hérnia Medular é feito por meio de ressonância magnética, onde é visto um deslocamento do conteúdo medular por um defeito dural, este deslocamento ocorre mais frequentemente para porção anterior da coluna.

Este relato demonstra o caso de uma paciente com uma apresentação clínica e anatômica atípica de HMI, que possui uma variação posicional da lesão, sendo esta de caráter lateral, fugindo do típico deslocamento ventral. O caso foi tratado cirurgicamente em nossa instituição, sendo necessários planejamento e abordagem diferenciados para o mesmo.

Os autores descrevem a técnica cirúrgica para esta variação da apresentação, bem como os achados clínicos, radiológicos, intraoperatórios e a evolução no acompanhamento pós-operatório.

\section{Apresentação do caso}

\section{Apresentação clínica e radiológica}

Paciente do sexo feminino, de 38 anos de idade, sem comorbidades, com queixa de parestesia no membro inferior esquerdo na região do quadríceps há 2 anos, evoluindo lentamente com piora progressiva nos últimos 6 meses, associado a fraqueza na perna esquerda, dificuldade de deambular e piora da parestesia. Nega trauma ou procedimento cirúrgico prévio. Ao exame físico, apresenta força motora grau IV no membro inferior esquerdo, proximal e distal, presença de hiperreflexia patelar e aquileu à esquerda, parestesia no quadríceps, sinal de Babinski presente e marcha claudicante no membro inferior esquerdo. Fugindo à apresentação típica da Síndrome de Brown-Sequard, já que apresentava alteração sensitiva e motora do mesmo lado e não apresentava alteração termo-álgica, apenas parestesia.

O estudo por RM da coluna torácica evidencia uma herniação medular ao nível de T9-T10 que se estende pelo neuroforame à esquerda (-Fig. 1), com saída do conteúdo medular por uma falha dural, com orifício de $12 \mathrm{~mm}$ de dimensão, para o interior do forame adjacente, englobando a raiz de T9 esquerda, ausência de coluna liquórica à esquerda e deformação medular, com significante herniação do funículo lateral esquerdo.

A abordagem cirúrgica foi indicada devido a uma combinação de fatores que incluem piora clínica, déficit motor e presença de mielopatia.

\section{Procedimento cirúrgico}

Como este caso apresenta uma herniação lateral pelo forame e engloba a raiz adjacente, foi optado por realizar uma artrectomia por via posterior, facilitando a abordagem ao forame a fim de reduzir o volume que se encontrava em sua maior parte foraminal; radiculotomia à esquerda; correção do defeito dural e instrumentação de T9-T10-T11, devido à instabilidade gerada pela ampla laminectomia e ressecção da faceta para evitar cifose segmentar.

O procedimento cirúrgico foi realizado sob anestesia geral e sob monitorização com potenciais evocados somatossensitivo e motor. O paciente foi posicionado em decúbito ventral horizontal com coxins pélvico e subescapular. 0 acesso cirúrgico foi feito por linha média posterior com dissecção da musculatura paraespinal.

Foi realizada ampla laminectomia do nível T9 ao nível T11 e facetectomia da articulação de T9-T10 à esquerda. Com esta ressecção foi possível a exposição e observação do saco dural e retração do mesmo para a esquerda. Assim, foi possível a visualização da massa com extensão para o neuroforame adjacente, demonstrada na - Fig. 2.

Foi realizada uma durotomia, aproximadamente $5 \mathrm{~cm}$, e reparo em ambas as bordas da dura-máter com fio nylon 3-0, com ampla exposição do conteúdo intradural, auxiliando a ressecção dos ligamentos denteados, exposição da medula e do conteúdo herniado, assim como visualização interna da falha dural. Após abertura dural, foi observado deslocamento medular com herniação lateral pelo orifício dural esquerdo,

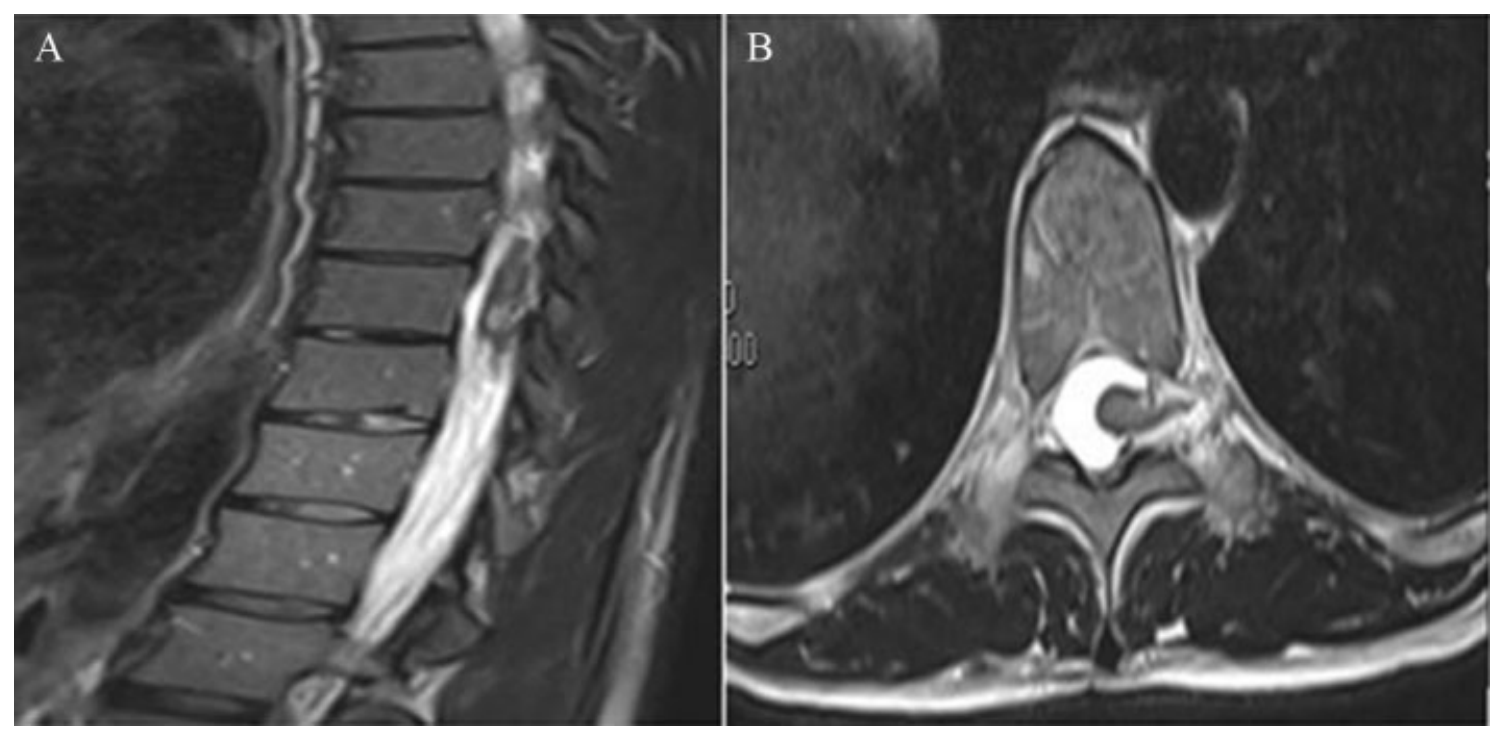

Fig. 1 Imagens sagital (A) e axial (B) de ressonância nuclear magnética ponderada em T2 demostram herniação medular ao nível de T9-T10 através de falha dural para o interior do neuroforame da raiz de T9 à esquerda. 


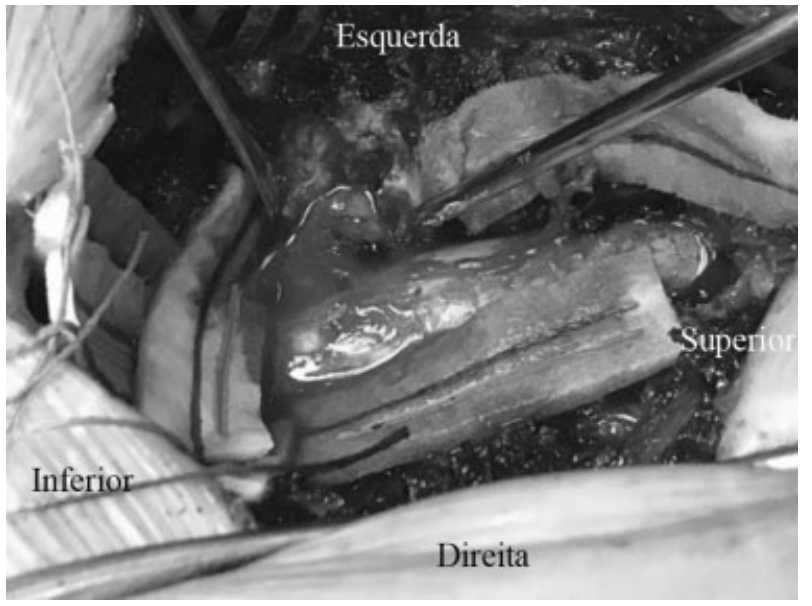

Fig. 2 Imagem intraoperatória do acesso posterior: laminectomia T9T10-T11, saco dural com presença de massa lateral com extensão ao neuroforame de T9

englobando a raiz emergente pelo forame ao nível T9, confirmando o diagnóstico de HMI (-Fig. 3).

Com auxílio de um dissector Rhoton e um microscópio, foi realizada microcirurgia com ressecção dos ligamentos denteados à esquerda, um nível acima e abaixo, possibilitando tração da medula e redução do volume herniado. Observada a ausência de plano para dissecção da raiz de T9, optou-se então pela sua radiculotomia. Foi realizado o fechamento do orifício com substituto dural e duroplastia com fio monofilamentar 5-0 (-Fig. 4).

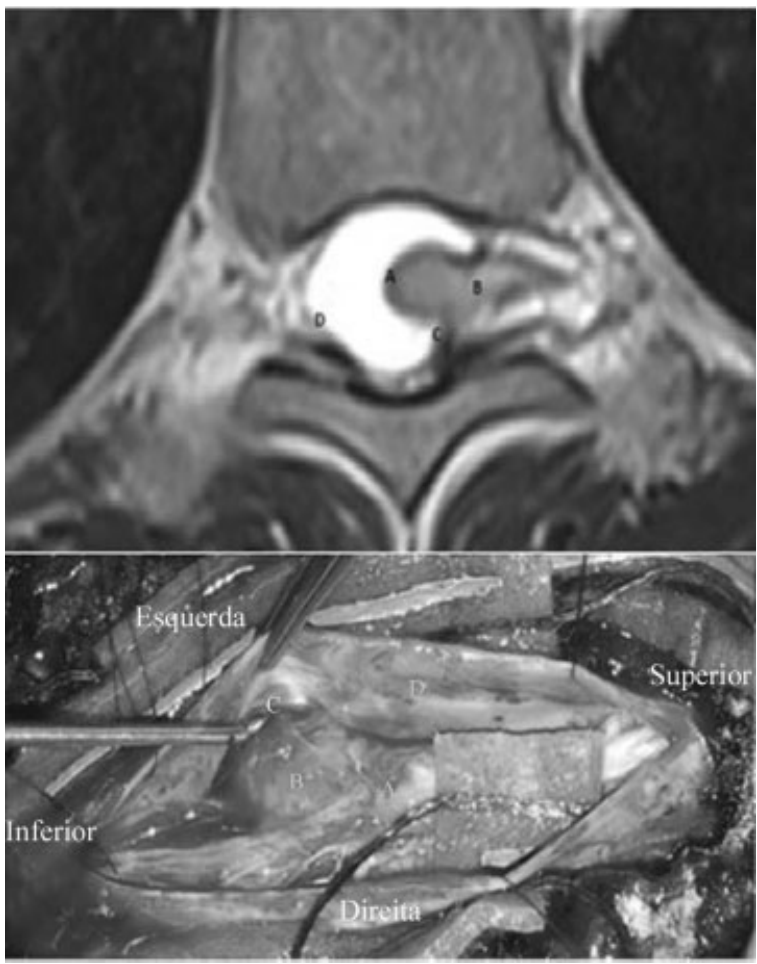

Fig. ${ }^{1}$ Imagem axial de tomografia computadorizada pré-operatória ao nível de T9 $\mathrm{e}^{2}$ fotografia intraoperatória pós-durotomia e resseção dos ligamentos denteados. Nas figuras são indicadas as seguintes estruturas anatômicas: (A) cordão medular, (B) herniação medular, (C) orifício dural e (D) dura-máter.

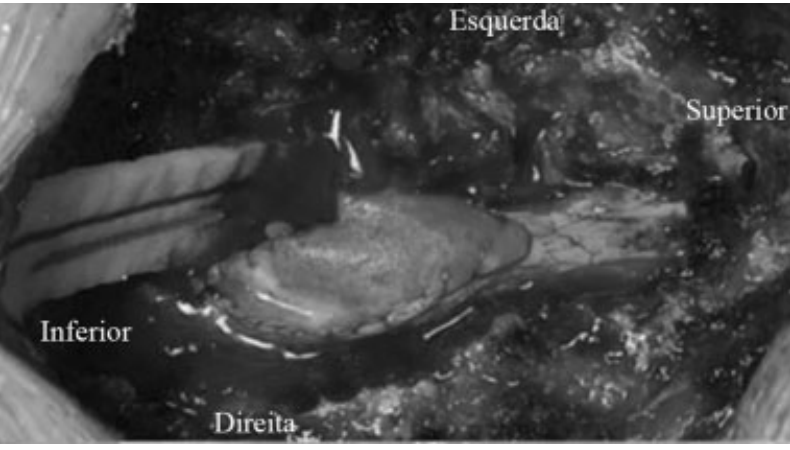

Fig. 4 Imagem intraoperatória mostrando fechamento com reconstrução (duroplastia) expansiva do espaço dural utilizando "wrap" com substituto.

Suplementação posterior e fixação pedicular devido a laminectomia de três vértebras consecutivas e artrectomia à esquerda, a fim de evitar uma possível evolução com acentuação da cifose. Para tal, foram utilizados seis parafusos transpediculares e duas hastes nos níveis de T9, T10 e T11 (-Fig. 5), de acordo com a técnica de Roy-Camille.

Os potenciais evocados mantiveram-se sem alterações durante o procedimento cirúrgico. A cirurgia teve duração total de 5 horas e perda sanguínea estimada em $800 \mathrm{~mL}$, a paciente foi encaminhada para a UTI imediatamente após a cirurgia.

No primeiro dia pós-operatório, a paciente apresentou piora da força e da sensibilidade em ambas as pernas. Por meio de novo estudo de imagem, diagnosticou-se um hematoma extradural na região dorsal, que exercia efeito de massa. A paciente foi então encaminhada para o centro cirúrgico para drenagem do hematoma e revisão da hemostasia. Após reabertura da sutura da pele e do tecido subcutâneo, foi feita a drenagem espontânea de um coágulo extradural de $50 \mathrm{~mL}$ de volume. Uma vez que realizadas a

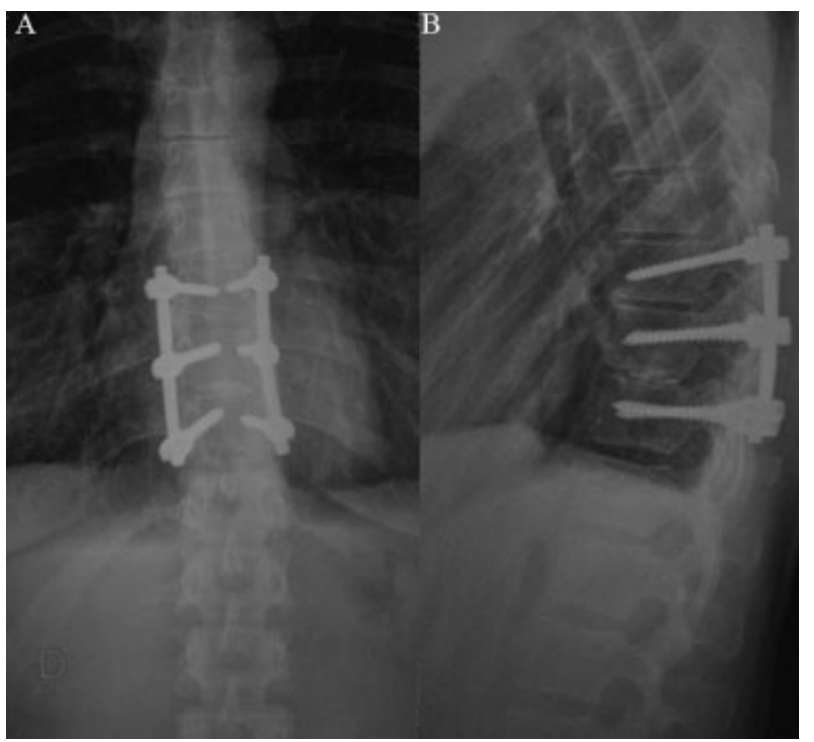

Fig. 5 Radiografias pós-operatórias evidenciando a instrumentação transpedicular em T9, T10 e T11. (A) Anteroposterior e (B) Lateral. 
drenagem e a coagulação de vasos do plexo epidural que apresentavam sangramento de pequena monta, a paciente foi então encaminhada novamente para a UTI, onde permaneceu por 1 dia.

Após a cirurgia de revisão, a paciente apresentou fístula liquórica de baixo débito, com resolução espontânea no terceiro dia após medidas clínicas e tratamento conservador com curativo compressivo, repouso no leito, decúbito zero e restrição de volume. A paciente recebeu alta no décimo dia pós-operatório após a internação, com resolução da fístula, ferida seca, sem queixas álgicas, mantendo pouca parestesia no trajeto torácico lateral esquerdo, melhora da força motora e da parestesia no membro inferior esquerdo, deambulando sem alterações.

A paciente mantém acompanhamento ambulatorial seriado, com reversão do déficit motor e parestesia no membro inferior esquerdo já no primeiro retorno em 15 dias e reversão da parestesia torácica lateral esquerda após 3 meses. A paciente manteve ainda hiperreflexia patelar $2+$ até o acompanhamento em 1 ano.

O estudo de imagem por ressonância magnética realizado 3 meses após o procedimento demonstrou redução do cordão medular e movimentação do líquido cefalorraquidiano na porção lateral esquerda do canal medular ( - Fig. 6).

\section{Discussão}

A HMI é uma causa rara de mielopatia torácica e muitas vezes sub diagnosticada. Sua fisiopatologia exata é desconhecida. Várias teorias foram levantadas: defeito ou erosão dural, secundária a cistos aracnoides, tumores, duplicidade da dura-máter, pós-operatório ou trauma. Sendo que a teoria mais aceita tem sido a descrita por Inoue et $\mathrm{al}^{3}{ }^{3}$ a qual classifica a HMI como um defeito dural congênito; segundo os autores, a HMI é mais comum no ápice da cifose torácica, entre os níveis T3-T7, e a herniação em quase todos os casos é voltada para a região ventral em direção ao disco. Essa teoria ainda propõe que a abertura de um orifício e a protrusão medular são facilitadas pela posição mais anterior da medula no ápice da cifose torácica associada à pulsação liquórica, aos movimentos de flexão e extensão, à expansão torácica durante a respiração e aos batimentos cardíacos. ${ }^{3}$ Com o advento de uma protrusão inicial, a pressão negativa do espaço extradural contribui para a herniação progressiva e o estrangulamento da medula nas margens do orifício, levando a uma isquemia medular evolutiva, afetando inicialmente o trato o espinotalâmico e posteriormente o trato córticoespinhal.

Como a localização mais frequente da herniação medular é anterior ou anterolateral, a apresentação clínica mais comum é a Síndrome de Brown-Sequárd progressiva, com cerca de 70\% dos casos tendo duração média dos sintomas de 40 meses. ${ }^{2,4,5}$ Outros pacientes podem apresentar paraparesia, alterações esfincterianas, distúrbios da sensibilidade, dorsalgia e dor no peito. Esta patologia afeta principalmente adultos de meia idade com predileção discreta pelo sexo feminino. ${ }^{6}$ Os achados clínicos e radiológicos contribuem para a decisão da abordagem cirúrgica, que apresenta uma alta taxa de melhora, principalmente dos sintomas motores, em $80 \%$, e sensitivos, em 35\%. ${ }^{7-9}$

A descrição de casos de HMI na literatura é esparsa. Summers e colaboradores, ${ }^{2}$ em uma revisão sistemática, identificaram 174 casos relatados na literatura, sendo que a grande maioria deles trata-se de herniações com padrão ventral ou ventro-lateral, e apenas um caso dorsal, mas não é descrito nenhum caso com herniação lateral pelo forame radicular, o que individualiza o caso relatado no presente trabalho. Nosso caso apresenta uma variação da localização da HMI, uma volumosa herniação do fúniculo lateral em conjunto com a raiz de T9 pelo forame esquerdo, obliterando a coluna liquórica lateral; por este motivo, associado a sinais clínicos de déficit motor e mielopatia, foi optado por abordagem cirúrgica.

Várias técnicas são descritas para o tratamento de HMI. Como a grande maioria das apresentações são de padrão ventral, o acesso dorsal é o mais utilizado. ${ }^{10}$ A estratégia cirúrgica deve ser individualizada para cada paciente. Porém,

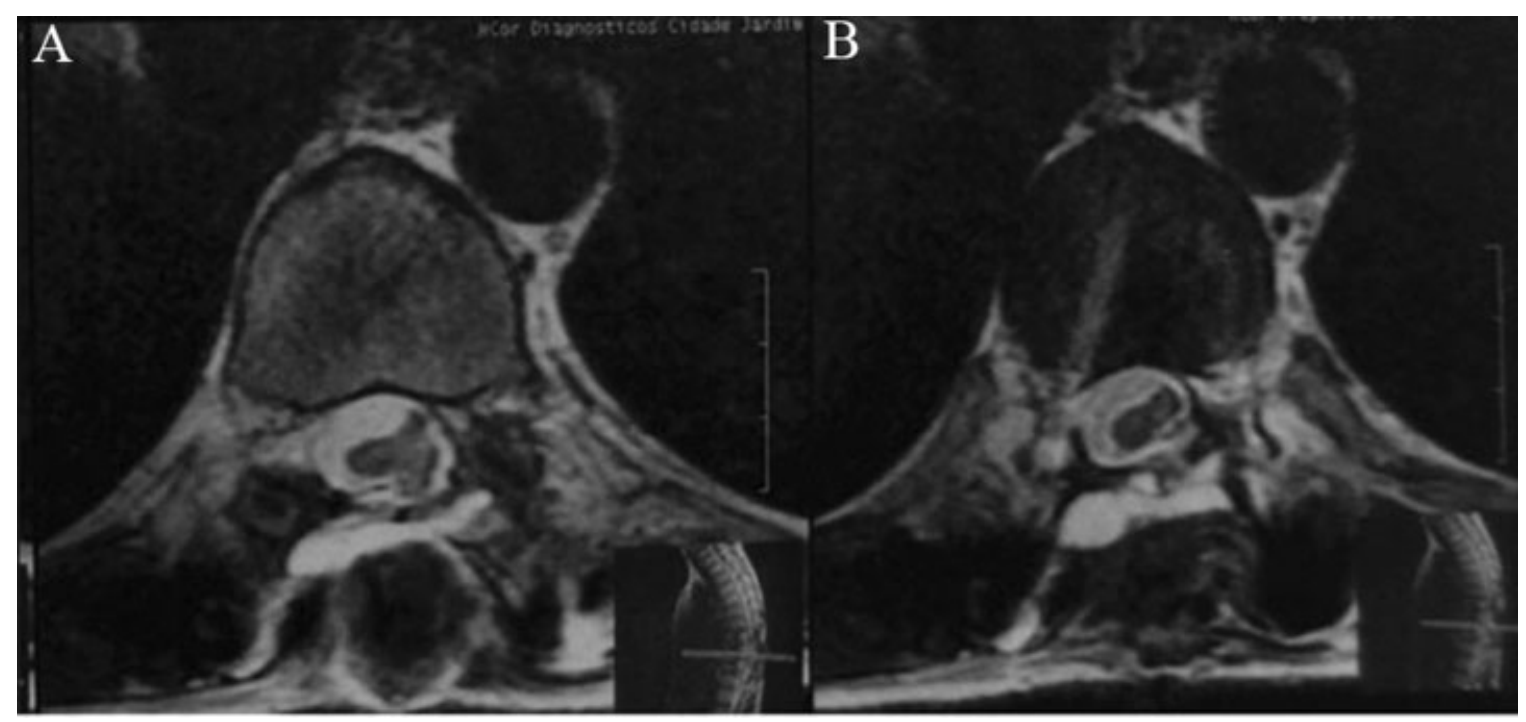

Fig. 6 Imagens axiais de ressonância nuclear magnética ponderada em T2 ao nível do disco intervertebral de T9-T10 após 3 meses de cirurgia. É possível notar a redução da hérnia medular foraminal esquerda. 
independente da causa ou da localização, a proposta básica será a redução do volume herniado mantendo a anatomia o mais próximo do normal, a fim de evitar recorrência e piora da função neurológica. ${ }^{11,12}$

Por se tratar de uma HMI lateral, algumas alterações no procedimento cirúrgico foram necessárias. $\mathrm{O}$ acesso se manteve posterior e mediano. Para chegar ao sítio da herniação entrando pelo forame, os cirurgiões tiveram que realizar uma artrectomia unilateral total de T9-T10, de forma a permitir visualização da raiz e facilitar na redução, já que a maior parte do conteúdo extradural encontrava-se foraminal. Como não existia plano de clivagem entre a medula e a raiz, não foi possível a dissecção e preservação da raiz, sendo optado pela radiculotomia desta estrutura para atingir a total redução do volume herniado.

Os casos descritos na literatura são em sua grande maioria de poucas séries, com relato um a três casos ou revisão da literatura, não sendo amplamente relatadas as complicações e intercorrências mais comuns. A piora do déficit motor e da mielopatia são as complicações mais temidas; além dessas, cifose secundária a laminectomia, infecção pós-operatória e fístula também podem ocorrer. A recorrência da herniação é extremamente rara, descrita em apenas um caso na literatura, com retorno da Síndrome de Brown-Sequard após 10 anos da cirurgia corretiva. ${ }^{10}$

Mesmo apresentando complicações pós-operatórias, a paciente descrita neste artigo evoluiu com melhora do déficit motor e da sensibilidade já no pós-operatório e no acompanhamento em 6 meses, com reversão do déficit motor e da parestesia no membro inferior esquerdo, marcha atípica, resolução da parestesia torácica esquerda, mantendo hiperreflexia patelar esquerda e com relato de melhora total dos sintomas iniciais.

\section{Conclusão}

A HMI é uma patologia rara, com variação tanto na localização da lesão quanto nos sintomas clínicos correspondentes. Neste artigo relatamos um caso atípico de HMI, nunca antes relatado na literatura, nos obrigando a programar uma abordagem cirúrgica diferenciada. Cabe ao cirurgião de coluna saber identificar e diagnosticar um caso de HMI, tendo em mente que esta pode ter diferentes apresentações clínicas e anatômicas. Sendo assim, a estratégia de manejo terapêutico e cirúrgico deve ser individualizado, e a abordagem cirúrgica, quando necessária, pode levar a resultados clínicos satisfatórios, com preservação da função neurológica através da correção da lesão envolvida.

Conflitos de interesse

Dr. Amaral reports personal fees from NuVasisve, outside the submitted work. Dr. Jensen reports personal fees from NuVasisve, outside the submitted work. Dr. Luiz Pimenta reports personal fees from NuVasive, outside the submitted work. All the other authors declare that they have no conflicts of interest to report.

\section{Referências}

1 White BD, Firth JL. Anterior spinal hernia: an increasingly recognised cause of thoracic cord dysfunction. J Neurol Neurosurg Psychiatry 1994;57(11):1433-1435

2 Summers JC, Balasubramani YV, Chan PCH, Rosenfeld JV. Idiopathic spinal cord herniation: Clinical review and report of three cases. Asian J Neurosurg 2013;8(02):97-105

3 Inoue T, Cohen-Gadol AA, Krauss WE. Low-pressure headaches and spinal cord herniation. Case report. J Neurosurg 2003;98(1, Suppl)93-95

4 Nakamura M, Fujiyoshi K, Tsuji O, et al. Long-term surgical outcomes of idiopathic spinal cord herniation. J Orthop Sci 2011;16 (04):347-351

5 Batzdorf U, Holly LT. Idiopathic thoracic spinal cord herniation: report of 10 patients and description of surgical approach. J Spinal Disord Tech 2012;25(03):157-162

6 Najjar MW, Baeesa SS, Lingawi SS. Idiopathic spinal cord herniation: a new theory of pathogenesis. Surg Neurol 2004;62(02): 161-170, discussion 170-171

7 Parmar H, Park P, Brahma B, Gandhi D. Imaging of idiopathic spinal cord herniation. Radiographics 2008;28(02):511-518

8 Rivas JJ, de la Lama A, Gonza Lez P, Ramos A, Zurdo M, Alday R. [Spontaneous spinal cord herniation]. Neurocir Astur Spain. Outubro de 2004;15(05):484-489

9 Sioutos P, Arbit E, Tsairis P, Gargan R. Spontaneous thoracic spinal cord herniation. A case report. Spine 1996;21(14):1710-1713

10 Selviaridis P, Balogiannis I, Foroglou N, Hatzisotiriou A, Patsalas I. Spontaneous spinal cord herniation: recurrence after 10 years. Spine J 2009;9(03):e17-e19

11 Borges LF, Zervas NT, Lehrich JR. Idiopathic spinal cord herniation: a treatable cause of the Brown-Sequard syndrome-case report. Neurosurgery 1995;36(05):1028-1032, discussion 1032-1033

12 Chaichana KL, Sciubba DM, Li KW, Gokaslan ZL. Surgical management of thoracic spinal cord herniation: technical consideration. J Spinal Disord Tech 2009;22(01):67-72 